\title{
A randomised controlled trial: effect of the meticulous nursing model on the treatment compliance and quality of life of patients with upper gastrointestinal bleeding
}

\author{
Hongyan Ai \\ Department of Gastroenterology, Linyi Central Hospital, Linyi, China \\ Correspondence to: Hongyan Ai. Department of Gastroenterology, Linyi Central Hospital, No.17 Jiangkang Road, Yishui, Linyi 276400, China. \\ Email: 1xfahy@163.com.
}

\begin{abstract}
Background: The purpose of this study was to analyze the effect of the meticulous nursing model on the treatment compliance and quality of life of patients with upper gastrointestinal bleeding (UGIB).

Methods: A total of 108 UGIB patients treated in Linyi Central Hospital from October 2018 to October 2019 were selected as the study subjects, and were randomly divided into a research group and reference group, with 54 cases in each group. The reference group received conventional nursing while the research group received meticulous nursing on this basis to compare the clinical intervention effect and the impact on quality of life in the 2 groups of patients.
\end{abstract}

Results: The Generic Quality of Life Inventory-74 (GQOLI-74) scores in the 2 groups of patients after intervention were significantly higher than those before intervention $(\mathrm{P}<0.001)$, and the score of the research group after intervention was significantly higher than that of the reference group $(\mathrm{P}<0.001)$. The Stanford Acute Stress Reaction Questionnaire (SASRQ) scores of the patients presented a trend opposite to GQOLI-74 $(\mathrm{P}<0.001)$. The number of fully satisfied cases in the research group was significantly higher than that in the reference group $(\mathrm{P}<0.05)$, while the number of dissatisfied cases was significantly lower than that in the reference group $(\mathrm{P}<0.05)$. The self-rating anxiety scale (SAS) scores in the 2 groups of patients after intervention were significantly lower than those before intervention $(\mathrm{P}<0.001)$, and the score of the research group after intervention was significantly lower than that of the reference group $(\mathrm{P}<0.001)$. The total clinical effective rate and treatment compliance of the research group were significantly higher than those of the reference group $(\mathrm{P}<0.05)$.

Conclusions: The meticulous nursing model can effectively improve the quality of life of UGIB patients, reduce the psychological stress response, and improve clinical treatment compliance and nursing satisfaction with a definite effect, making it worthy of promotion and application.

Trial Registration: Chinese Clinical Trial Registry ChiCTR2100048735.

Keywords: Meticulous nursing; upper gastrointestinal bleeding (UGIB); treatment compliance; life quality

Submitted Apr 20, 2021. Accepted for publication Aug 17, 2021.

doi: 10.21037/apm-21-1283

View this article at: https://dx.doi.org/10.21037/apm-21-1283

\section{Introduction}

Upper gastrointestinal bleeding (UGIB), a common gastrointestinal disease, usually refers to gastrointestinal bleeding above the Treitz, occurring at the stomach, esophagus, duodenum, and biliary and pancreatic regions (1-3). Epidemiological studies have found that the mortality rate of this disease is as high as $25-30 \%$, and mainly occurs in adults. According to the different types of the disease, it can be specifically divided into chronic concealed hemorrhage, chronic revealed hemorrhage, and acute massive hemorrhage, with the characteristics of acute onset and rapid progression. If patients are not treated in 
time, their lives will be endangered (4-6). Since clinical studies have confirmed that gastrointestinal ulcer is the main cause of UGIB, active treatment of gastrointestinal ulcers can effectively prevent UGIB. Due to its acute onset, some patients suffer from massive hematochezia and hematemesis, which easily lead to fear and anxiety of different degrees and psychological stress reactions, affecting clinical treatment (7). The psychological stress response is an individual non-specific response caused by various stressors, including increased corticosteroid secretion, increased blood glucose and blood pressure, and increased heart rate, which will affect the clinical treatment of patients to a certain extent. Therefore, in addition to emergency treatment, effective clinical nursing measures should be taken for UGIB patients, which is of great significance for improving negative emotions, improving treatment compliance, and reducing mortality (8-10). The meticulous nursing model centers on patients, and further optimizes and subdivides the nursing process, thereby truly grasping the inner demands of patients. While controlling bleeding, this model alleviates negative emotions, achieving a comprehensive, detailed, and thoughtful nursing process, so as to provide better inpatient nursing services and improved patient satisfaction (11). Based on this, this paper aimed to further study the effect of the meticulous nursing model on the treatment compliance and quality of life of UGIB patients. We present the following article in accordance with the CONSORT reporting checklist (available at https:// dx.doi.org/10.21037/apm-21-1283).

\section{Methods}

\section{General information}

A total of 108 UGIB patients treated in Linyi Central Hospital from October 2018 to October 2019 were selected as the subjects for two-parallel study, and were randomly divided into a research group and reference group, with 54 cases in each group. The allocation ratio is $1: 1$. The study was conducted in accordance with the Declaration of Helsinki (as revised in 2013). The study was approved by ethics board of Linyi Central Hospital (No: 20181005) and informed consent was taken from all individual participants.

\section{Inclusion criteria}

(I) Patients met the diagnostic criteria for UGIB; (II) patients had complete clinical data; (III) patients were in a stable condition after treatment; (IV) this study was approved by the hospital ethics committee, and the patients and their families knew the content of this experimental study, and signed the informed consent.

\section{Exclusion criteria}

(I) Patients had mental disorders and other cognitive disorders or communication disorders; (II) patients had organic lesions such as those of the brain, heart, and liver; (III) patients had a history of gastrointestinal bleeding; (IV) patients refused to cooperate with the experiment.

\section{Study methods}

Conventional nursing was carried out in the reference group through regularly measuring indicators such as routine blood, liver function, and feces tests of the patients, instructing patients to take their medications on time, implementing dietary interventions, and formulating detailed treatment plans after observation of the disease progression of the patients.

The research group received meticulous nursing on the basis of conventional nursing, and additional processes as follows: (I) the ward was disinfected regularly every day to keep the ward clean and tidy. Fresh and soothing music was played with the approval of patients or family members to eliminate the tension and anxiety of patients and relax them physically and mentally. (II) The patients were instructed to rest on time and maintain adequate sleep time. The nursing staff actively communicated with patients, listened to them patiently and carefully, and provided psychological counseling in time. The staff also informed patients of the relevant UGIB knowledge so that patients correctly understood their own disease and had more courage and confidence to overcome the disease, improving treatment compliance. The daily visits of family members were recorded to ensure that each patient was accompanied by family members during hospitalization. The patients were informed of the importance of family company to reduce their loneliness and make them feel warmth from their family. (III) The specific lifestyles of patients and their families were investigated to formulate detailed life plans for patients, and publicize risk factors. (IV) Exercise prescriptions were formulated based on the clinical condition and physical fitness of patients, maintaining existing limb motor function, and preventing the occurrence 
of "disuse". (V) According to patients' conditions, the staff formulated specific medication schedules, supervised and recorded patients' medications, and informed patients and their families of medication precautions.

\section{Observation indexes}

General data of patients in both groups were analyzed and compared, including gender, age, body mass index (BMI), smoking history, drinking history, marital status, and UGIB volume.

The Generic Quality of Life Inventory-74 (GQOLI-74) (primary outcome) (12) was used to evaluate the quality of life in both groups of patients before and after intervention. The scale was scored from 4 scoring factors including psychological function, physical function, social function, and material life status, with a total score of 100 points. The higher the score, the better the quality of life.

The Stanford Acute Stress Reaction Questionnaire (SASRQ) (primary outcome) (13) was used to evaluate the psychological stress responses in both groups of patients before and after intervention, with a total score of 150 points. The higher the score, the stronger the psychological stress response of the patients.

The patient clinical satisfaction questionnaire prepared by the department was used, and the patients filled it in truthfully (primary outcome). According to the degree of clinical nursing satisfaction, the questionnaire was divided into fully satisfied, satisfied, needing improvement, and dissatisfied.

The self-rating anxiety scale (SAS) (primary outcome) (14) was used to evaluate the anxiety level in both groups of patients before and after intervention. With a total score of 100 points, this scale was divided into normal ( $<50$ points), mild anxiety (50-59 points), moderate anxiety (60-69 points), and severe anxiety (>69 points).

For efficacy judgments (primary outcome), markedly effective: blood loss was well controlled after intervention with no bleeding, and symptoms of hematochezia and hematemesis ceased. After 48 hours, occult blood was negative for 3 consecutive examinations. Effective: the bleeding condition was basically controlled, and symptoms of hematochezia and hematemesis ceased. After 72 hours, occult blood was negative for 3 consecutive examinations. Ineffective: The bleeding condition was not effectively controlled, and the related symptoms were not improved or even aggravated. Three consecutive occult blood tests were positive and invalid. Among them, total effective rate $=$ markedly effective rate + effective rate.

The self-prepared evaluation form of patient clinical treatment compliance (secondary outcome) was used to evaluate treatment compliance in the 2 groups of patients after intervention. With a total score of 100 points, the scale was divided into complete compliance ( $\geq 85$ points), partial compliance (75-84 points), and noncompliance ( $\leq 74$ points).

\section{Statistical analysis}

The experimental data were statistically analyzed and processed by SPSS 20.0 software. GraphPad Prism 6 (GraphPad Software, San Diego, USA) was used for image rendering of the data. Count data were tested by the $x^{2}$ test and expressed as $\mathrm{n}(\%)$. Measurement data were measured by the $t$ test and expressed as $\bar{x} \pm$ s. The difference was statistically significant when $\mathrm{P}<0.05$.

\section{Results}

\section{Comparison of general data in the 2 groups of patients}

As was shown in Figure 1, 158 patients were included in this study, and were divided into research group and reference group. There were no remarkable differences in gender ratio, age, BMI value, smoking and drinking histories, marital status, and UGIB volume between the 2 groups of patients $(\mathrm{P}>0.05)$, as shown in Table 1.

\section{Comparison of quality of life scores between of patients}

Compared with the GQOLI-74 scores of patients before intervention, the scores of all patients after intervention increased obviously $(\mathrm{P}<0.05)$, and the score of the research group after intervention was significantly higher than that of the reference group $(\mathrm{P}<0.05)$, as shown in Figure 2.

\section{Comparison of SASRQ scores before and after intervention between the 2 groups of patients}

The SASRQ scores in the 2 groups of patients after intervention were significantly lower than those before intervention $(\mathrm{P}<0.05)$, and the score of the research group after intervention was significantly lower than that of the reference group $(\mathrm{P}<0.05)$, as shown in Figure 3. 


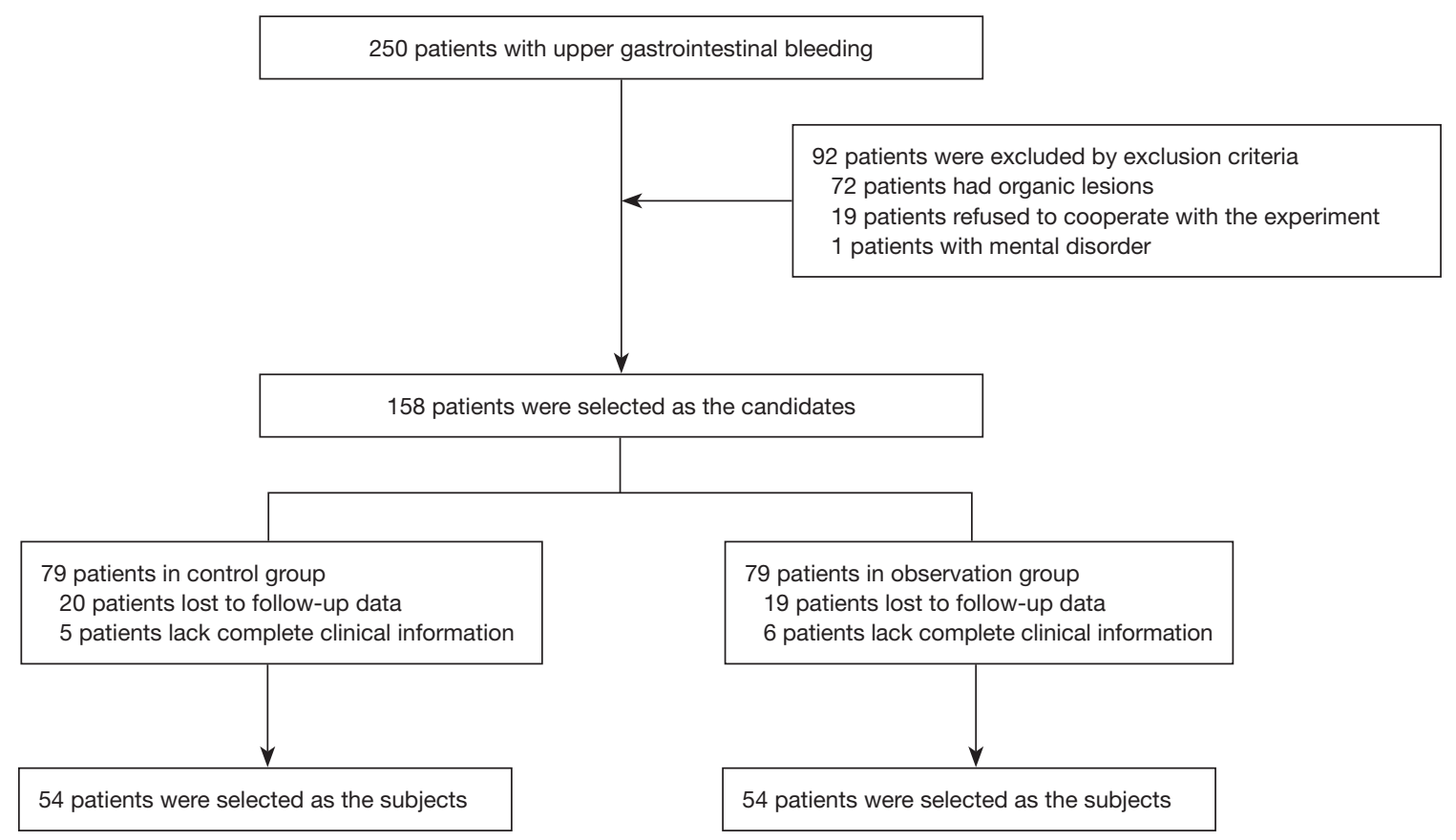

Figure 1 The flow diagram of subject selection.

Table 1 Comparison of general data in the 2 groups of patients [n (\%), $\bar{x} \pm$ s]

\begin{tabular}{|c|c|c|c|c|}
\hline Items & Research group $(n=54)$ & Reference group $(n=54)$ & $\chi^{2} / t$ & $P$ \\
\hline Male & 29 (53.70\%) & 31 (57.41\%) & & \\
\hline Female & $25(46.30 \%)$ & $23(42.59 \%)$ & & \\
\hline Average age (years old) & $42.35 \pm 3.46$ & $42.38 \pm 3.42$ & 0.045 & 0.964 \\
\hline Smoking history & & & 0.152 & 0.697 \\
\hline No & 32 (59.26\%) & 30 (55.56\%) & & \\
\hline Yes & $22(40.74 \%)$ & $24(44.44 \%)$ & & \\
\hline Drinking history & & & 0.172 & 0.679 \\
\hline Marital status & & & 0.101 & 0.750 \\
\hline Unmarried & $48(88.89 \%)$ & $49(90.74 \%)$ & & \\
\hline Married & $6(11.11 \%)$ & $5(9.26 \%)$ & & \\
\hline $\begin{array}{l}\text { Average upper gastrointestinal } \\
\text { bleeding volume }(\mathrm{mL})\end{array}$ & $746.89 \pm 102.35$ & $747.03 \pm 102.41$ & 0.007 & 0.994 \\
\hline
\end{tabular}

BMI, body mass index. 


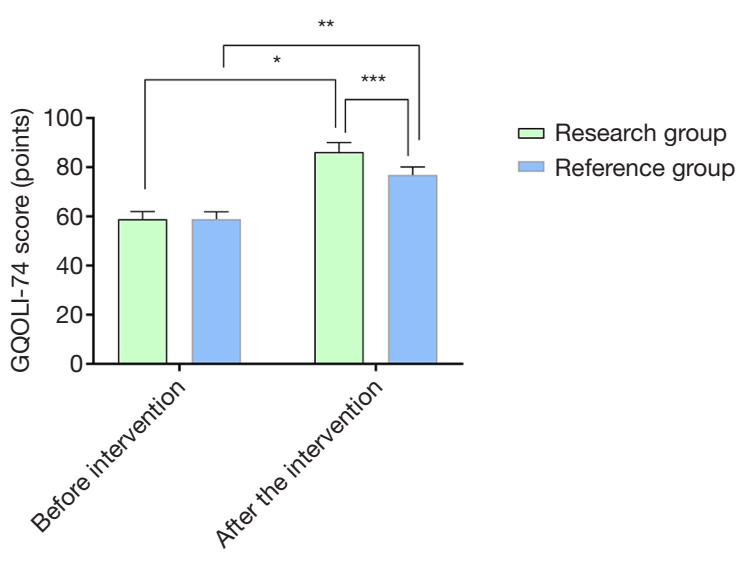

Figure 2 Comparison of quality of life scores before and after intervention between the 2 groups of patients. The abscissa represents the stages of intervention, and the ordinate represents GQOLI-74 score (points). The GQOLI-74 scores of patients in the research group before and after intervention were $56.68 \pm 4.37$ points and $83.54 \pm 5.36$ points, respectively. The GQOLI-74 scores of patients in the reference group before and after intervention were $56.70 \pm 4.31$ points and $74.62 \pm 4.55$ points, respectively. * indicated that there was a significant difference in GQOLI-74 scores of all patients before and after intervention $(\mathrm{t}=31.287$, $\mathrm{P}=0.000) ; * *$ indicated that there was a significant difference in GQOLI-74 scores in the reference group before and after intervention ( $\mathrm{t}=21.011, \mathrm{P}=0.000)$; ${ }^{* *}$ indicated that there was a significant difference in GQOLI-74 scores between the 2 groups after intervention ( $\mathrm{t}=10.187, \mathrm{P}=0.000)$. GQOLI-74, Generic Quality of Life Inventory-74.

\section{Comparison of the satisfaction of patients on clinical nursing}

There were no significant differences in the number of patients who were satisfied and the number needing improvement between the 2 groups of patients $(P>0.05)$. The number of fully satisfied cases in the research group was significantly higher than that in the reference group $(\mathrm{P}<0.05)$, while the number of dissatisfied cases was obviously lower than that in the reference group (Table $2, \mathrm{P}<0.05)$.

\section{Comparison of the SAS scores of patients}

Compared with the SAS scores of patients before intervention, the scores of the patients after intervention decreased obviously $(\mathrm{P}<0.05)$, and the score of the research group after intervention was remarkably lower than that of the reference group (Figure 4, $\mathrm{P}<0.05$ ).

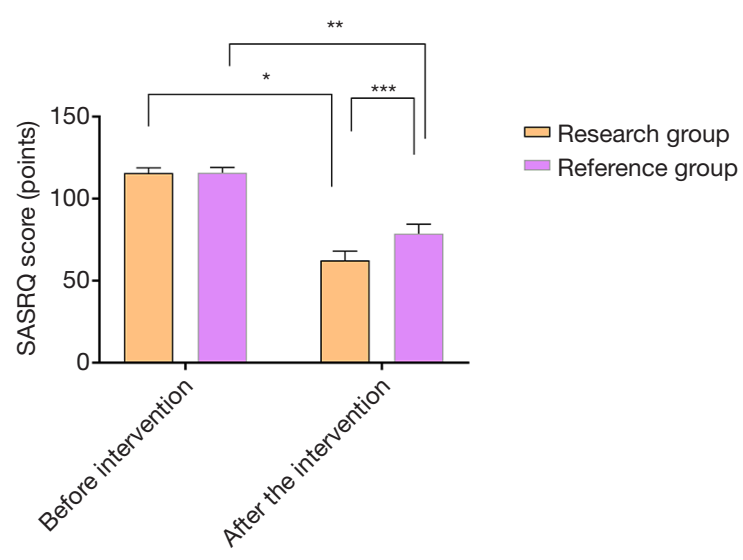

Figure 3 Comparison of SASRQ scores between the 2 groups of patients before and after intervention. The abscissa represents before intervention and after intervention, and the ordinate indicates SASRQ score (points). The SASRQ scores of patients in the research group before and after intervention were $113.56 \pm 4.37$ points and $58.32 \pm 8.12$ points, respectively. The SASRQ scores of patients in the reference group before and after intervention were $113.49 \pm 4.58$ points and $74.69 \pm 8.07$ points, respectively. * indicated that there was a significant difference in SASRQ scores of all patients before and after intervention ( $\mathrm{t}=40.021, \mathrm{P}=0.000)$; ** indicated that there was a significant difference in SASRQ scores in the reference group before and after intervention $(t=30.727$, $\mathrm{P}=0.000) ;{ }^{* *}$ indicated that there was a significant difference in SASRQ scores between the 2 groups after intervention $(t=10.508$, $\mathrm{P}=0.000)$. SASRQ, Stanford Acute Stress Reaction Questionnaire.

\section{Comparison of clinical efficacy between the 2 groups of patients}

After nursing intervention, the total clinical effective rate of the research group was remarkably higher than that of the reference group (Table 3, $\mathrm{P}<0.05$ ).

\section{Comparison of treatment compliance between the 2 groups of patients}

After nursing intervention, the treatment compliance of the research group was remarkably higher than that of the reference group (Table 4, $\mathrm{P}<0.05$ ).

\section{Discussion}

Hematemesis and melanosis are typical clinical manifestations of UGIB diseases, and the severity of 
Table 2 Comparison of clinical nursing satisfaction between the 2 groups of patients [n (\%)]

\begin{tabular}{|c|c|c|c|c|}
\hline Degree of satisfaction & Research group $(n=54)$ & Reference group $(n=54)$ & $\chi^{2}$ & $P$ \\
\hline Satisfied & $14(25.93 \%)$ & $14(25.93 \%)$ & 0.000 & 1.000 \\
\hline Needing improvement & 5 (9.26\%) & $9(16.67 \%)$ & 1.313 & 0.252 \\
\hline Dissatisfied & $3(5.56 \%)$ & $12(22.22 \%)$ & 6.271 & 0.012 \\
\hline
\end{tabular}

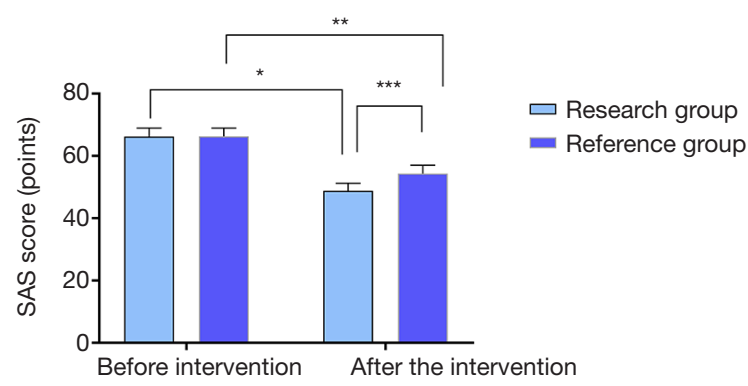

Figure 4 Comparison of SAS scores between the 2 groups of patients before and after intervention $(\bar{x} \pm \mathrm{s})$. The abscissa represents before intervention and after intervention, and the ordinate indicates SAS score (points). The SAS scores of patients in the research group before and after intervention were $64.32 \pm 3.84$ points and $47.16 \pm 3.35$ points, respectively. The SAS scores of patients in the reference group before and after intervention were $64.36 \pm 3.82$ points and $52.47 \pm 3.76$ points, respectively. ${ }^{*}$ indicated that there was a significant difference in SAS scores in the research group before and after intervention $(\mathrm{t}=24.745, \mathrm{P}=0.000)$; ${ }^{*}$ indicated that there was a significant difference in SAS scores in the reference group before and after intervention $(\mathrm{t}=16.301, \mathrm{P}=0.000)$; ${ }^{* *}$ indicated that there was a significant difference in SAS scores between the 2 groups after intervention $(\mathrm{t}=7.748, \mathrm{P}=0.000)$. SAS, self-rating anxiety scale.

Table 3 Comparison of clinical efficacy between the 2 groups of patients [n (\%)]

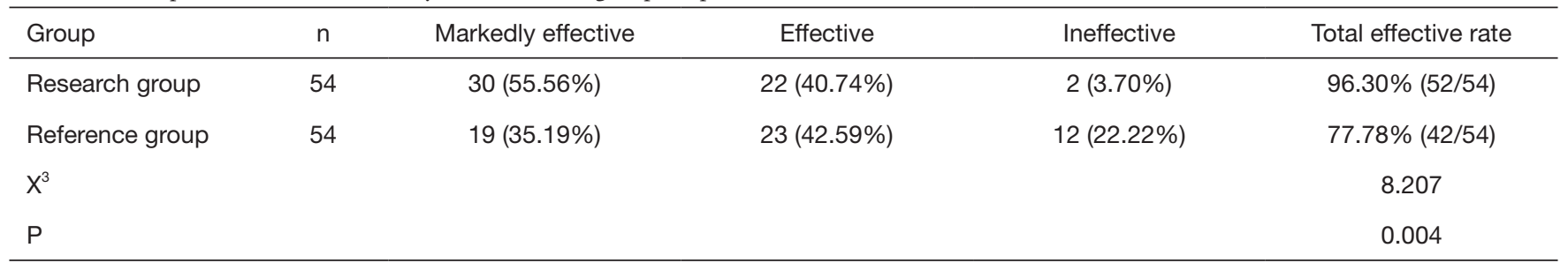

Table 4 Comparison of treatment compliance between the 2 groups of patients [n (\%)]

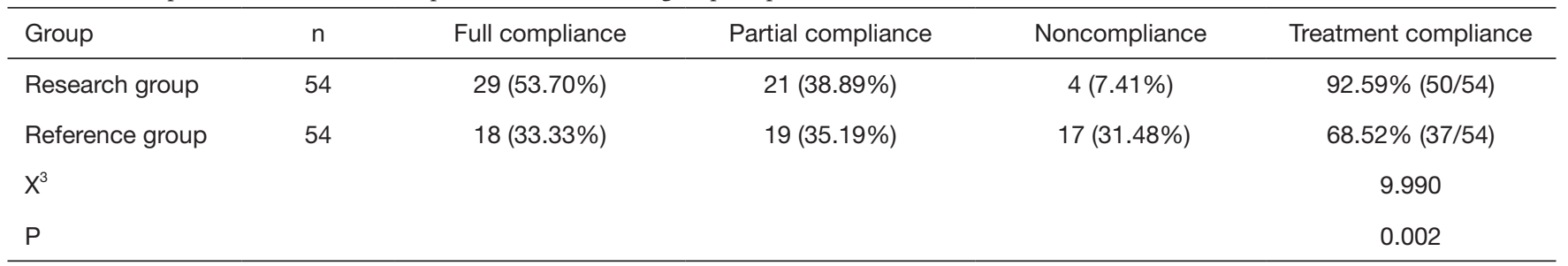


symptoms is related to the amount of blood loss and bleeding speed. Excessive blood loss will cause dizziness, fatigue, chills, and other symptoms, as well as shock, which can endanger life (15-17). Most of the bleeding symptoms in mild patients disappear by themselves, and effective hemostatic measures should be taken if they do not disappear by themselves. Antishock treatment and rapid blood volume supplementation should be put first in the rescue process. On this basis, targeted treatment measures should be taken according to the different types of UGIB (18-20). Most patients have a significant psychological burden due to obvious clinical symptoms, which makes clinical diagnosis and treatment difficult and creates higher requirements for the work of the medical staff. The meticulous nursing model grasps the daily lifestyles of UGIB patients through optimizing the nursing process, providing a basis for doctors to make accurate judgments. This nursing model can alleviate patients' negative emotions and eliminate fear and depression generated during the treatment process to establish treatment courage and confidence, improving treatment compliance $(21,22)$. This study found that after the patients of the research group received the meticulous nursing model on the basis of conventional nursing, the SAS score of the research group after intervention was significantly lower than that of the reference group. By keeping the ward environment clean and comfortable, and listening to beautiful and soothing music, the patients' negative emotions were stabilized to improve their internal anxiety. Psychologists believe that music, as a method to reduce stress, can stabilize anxiety and treat psychological disorders to a certain extent. In addition, music can also stimulate brain activity and brain cells, and can improve sleep quality (23). The results of this study are similar to the research results of Kiran et al. (24) who pointed out in their article that after patients with chronic revealed hemorrhage in upper gastrointestinal tract received meticulous nursing, the SAS score of patients $(47.37 \pm 2.47)$ after intervention was significantly lower than $(54.28 \pm 2.65)$ of the control group, indicating that meticulous nursing can effectively improve the anxiety of patients and promote treatment. Patient compliance (treatment compliance), also known as compliance, means that patients are treated according to the doctor's regulations (25). This study confirmed that the treatment compliance of patients in the research group after meticulous nursing intervention was significantly higher than that of the reference group. This may be because the nursing model was patientcentered, providing a comfortable treatment environment for patients, and informing them of disease-related knowledge to eliminate their incorrect cognition, establish treatment confidence, and effectively improve their treatment compliance. In addition, rational drug use and various physical examinations are also important measures to prevent the recurrence of UGIB. This study also had deficiencies, such as the small number of selected samples and the lack of exploration on the long-term efficacy of the nursing intervention, leading to insufficient accuracy of the research results. Therefore, it is necessary to increase the number of samples, prolong the follow-up time, and improve the accuracy and scientificity of experiments in the future.

In conclusion, the application of the meticulous nursing model in the clinical nursing of UGIB patients can effectively improve their quality of life, reduce the psychological stress response, and improve nursing satisfaction and treatment compliance with a definite effect, making it worthy of promotion and application.

\section{Acknowledgments}

Funding: None.

\section{Footnote}

Reporting Checklist: The author has completed the CONSORT reporting checklist. Available at https://dx.doi. org/10.21037/apm-21-1283

Trial Protocol: Available at https://dx.doi.org/10.21037/apm21-1283

Data Sharing Statement: Available at https://dx.doi. org/10.21037/apm-21-1283

Conflicts of Interest: The author has completed the ICMJE uniform disclosure form (available at https://dx.doi. org/10.21037/apm-21-1283). The author has no conflicts of interest to declare.

Ethical Statement: The author is accountable for all aspects of the work in ensuring that questions related to the accuracy or integrity of any part of the work are appropriately investigated and resolved. The study was conducted in accordance with the Declaration of Helsinki (as revised in 2013). The study was approved by ethics board of Linyi Central Hospital (No: 20181005) and informed 
consent was taken from all individual participants.

Open Access Statement: This is an Open Access article distributed in accordance with the Creative Commons Attribution-NonCommercial-NoDerivs 4.0 International License (CC BY-NC-ND 4.0), which permits the noncommercial replication and distribution of the article with the strict proviso that no changes or edits are made and the original work is properly cited (including links to both the formal publication through the relevant DOI and the license). See: https://creativecommons.org/ licenses/by-nc-nd/4.0/.

\section{References}

1. Afifi ANAM, Powerski M, Jechorek D, et al. Radiationinduced damage in the upper gastrointestinal tract: clinical presentation, diagnostic tests and treatment options. Best Pract Res Clin Gastroenterol 2020;4849:101711.

2. Okwara CJ, Gulati R, Rustagi T, et al. Upper Gastrointestinal Bleeding of Unusual Causation. Dig Dis Sci 2018;63:2541-6.

3. Abbass A, Khalid S, Boppana V, et al. Giant Gastric Ulcers: An Unusual Culprit. Dig Dis Sci 2020;65:2811-7.

4. Shi X, Yu S, Wang F, et al. A gastrointestinal stromal tumor with acute bleeding: Management and nursing. Medicine (Baltimore) 2018;97:e9874.

5. Liu YH, Lo EYJ, Lee KF, et al. An unusual cause of upper gastrointestinal bleeding due to recurrent hepatocellular carcinoma: A case report. Int J Surg Case Rep 2020;70:83-6.

6. Hicks N. Haemorrhagic cholecystitis: an unusual cause of upper gastrointestinal bleeding. BMJ Case Rep 2014;2014:bcr2013202437.

7. Guddati H, Andrade C, Muscarella P, et al. An unusual cause of massive upper gastrointestinal bleedinggastric mucormycosis. Oxf Med Case Reports 2019;2019:omy135.

8. Han S, Brauer BC, Scott FI. A Heart-Stopping Cause of Upper Gastrointestinal Bleeding. Gastroenterology 2018;155:983-4.

9. Cañamares-Orbís P, Chan FKL. Endoscopic management of nonvariceal upper gastrointestinal bleeding. Best Pract Res Clin Gastroenterol 2019;42-43:101608.

10. Awadalla MS, Desimone M, Wassef W. Updates on management of nonvariceal upper gastrointestinal bleeding. Curr Opin Gastroenterol 2019;35:517-23.
11. Twum-Barimah E, Abdelgadir I, Gordon M, et al. Systematic review with meta-analysis: the efficacy of tranexamic acid in upper gastrointestinal bleeding. Aliment Pharmacol Ther 2020;51:1004-13.

12. Baba Y, Kawano S, Kono Y, et al. Clinical Characteristics and Risk Factors for Rebleeding in Patients with Obscure Gastrointestinal Bleeding. Intern Med 2020;59:1345-50.

13. Yang QY, Ouyang J, Yang JD. Sepsis as an important risk factor for gastrointestinal bleeding in acute coronary syndrome patients: Two case reports. Medicine (Baltimore) 2018;97:e12273.

14. Lee PL, Yang KS, Tsai HW, et al. Tranexamic acid for gastrointestinal bleeding: A systematic review with metaanalysis of randomized clinical trials. Am J Emerg Med 2021;45:269-79.

15. Beyda R, Johari D. Tranexamic Acid for Upper Gastrointestinal Bleeding. Acad Emerg Med 2019;26:1181-2.

16. Bronswijk M, Bossuyt P, Christiaens $\mathrm{P}$, et al. Upper gastrointestinal bleeding due to Gurvits' syndrome. Dig Liver Dis 2019;51:1347.

17. Poddar U. Diagnostic and therapeutic approach to upper gastrointestinal bleeding. Paediatr Int Child Health 2019;39:18-22.

18. Anchu AC, Mohsina S, Sureshkumar S, et al. External validation of scoring systems in risk stratification of upper gastrointestinal bleeding. Indian J Gastroenterol 2017;36:105-12.

19. Chen YC, Chuang CJ, Hsiao KY, et al. Massive transfusion in upper gastrointestinal bleeding: a new scoring system. Ann Med 2019;51:224-31.

20. AbdAllah M. ANCYLOSTOMIASIS CAUSING UPPER GASTROINTESTINAL BLEEDING: REAL-TIME ENDOSCOPIC PICTURES. Gastroenterol Nurs 2019;42:179-80.

21. Irving EL, Sivak AM, Spafford MM. "I can see fine": patient knowledge of eye care. Ophthalmic Physiol Opt 2018;38:422-31.

22. Giardina S, Montinari MR. The wounded maternity in the fine arts. J Matern Fetal Neonatal Med 2018;31:2737-41.

23. Ray WA, Chung CP, Murray KT, et al. Association of Oral Anticoagulants and Proton Pump Inhibitor Cotherapy With Hospitalization for Upper Gastrointestinal Tract Bleeding. JAMA 2018;320:2221-30.

24. Gaspar FW, Maddalena R, Williams J, et al. Ultrafine, fine, and black carbon particle concentrations in California 
child-care facilities. Indoor Air 2018;28:102-11.

25. Saadat H, Bissonnette B, Tumin D, et al. Effects of partial sleep deprivation on reaction time in anesthesiologists.

Cite this article as: Ai H. A randomised controlled trial: effect of the meticulous nursing model on the treatment compliance and quality of life of patients with upper gastrointestinal bleeding. Ann Palliat Med 2021;10(8):8737-8745. doi: 10.21037/apm-21-1283
Paediatr Anaesth 2017;27:358-62.

(English Language Editor: C. Betlazar-Maseh) 